\author{
International Journal of Innovative Research in \\ Electrical, Electronics, Instrumentation and Control Engineering
}

Vol. 6, Issue 11, November 2018

\title{
Improvements for High-Precision Differential Microscopy Technique Based on Position Sensitive Detectors
}

\author{
Chen Liu ${ }^{1}$, Teo Zeng ${ }^{2}$, Beng $\mathrm{Wei}^{3}$, Kanai Ogale ${ }^{4}$, Lim Zhang ${ }^{5}$ \\ Department of Integrative Sciences and Engineering, National University of Singapore 1,2,3,4,5 $^{\text {. }}$
}

\begin{abstract}
A microscopy system has been introduced based on a Position Sensitive Detector (PSD). In our previous research, the geometric error factors that are caused by the pincushion-type distortion of these sensors were investigated and were addressed to significantly reduce Signal to Noise Ratio (SNR) in PSD and the microscopy system. The algorithm used for the microscopy system can be also further improved to achieve a high-precision system based on localized differential method. This approach was implemented in this research resulting in significant improvement in the precision of the microscopy system.
\end{abstract}

Keywords: Position Sensitive Detector; Localized differential method; Microscopy systems; PSD; Sensor calibration

\section{INTRODUCTION}

The Optical Position Sensor (OPS) can include a wide range of devices. One of them is the position sensitive detector or PSD. This sensor has been used in many applications including the atomic force microscopy [1]. This shows the range of applications that this sensor can cover. Still providing a precise measurement using the PSD is one of the challenges that is addresses in many researches.

The most commonly used PSD is the Lateral Effect PSD (LEP). Despite dedicated and carefully optimized technology, the position sensing accuracy and sensitivity of LEPs are poorer than those of photodetector arrays such as CCDs [2]. This has led investigations in using the CMOS monolithic calibrated position-sensitive detectors [3]. Another application of these PSD in techniques including Laser Scanning Microscopy [4][5].

There are variations of Laser Scanning Microscopy than include CCDs [6] or by using PSDs [7]. Using the Lateral Photo effect is the common practice among these approaches [8]. In any case, these techniques are limited to 2D scanning and there very few successful approaches that include 3D reconstruction of the scanning although the original scanning was done in $2 \mathrm{D}[9]$.

There are different methods suggested for performing measurements on the PSD. One of them is using the pulse amplitude modulation scheme [10]. The other technique is by using a local scanning of the object on the PSD [11][12]. Similarly, another approach was proposed by implementing a system for differential phase measurement [13]. The most interesting approach in this regard is the development of a LPS sensor system where PSD sensors are used as IR signal detectors [14].

Solving the problem of non-linearity of the PSD is the most important factor in improving the accuracy of measurements in these techniques. Song et al improved the non-linearity of the PSD significantly [15] but this is still an ongoing challenge. While there are approaches to enhance the measurement accuracy of the PSD, but still the SNR problem that causes the most important and boldest effect on the precision measurement of the PSD is left to be solved. The proposed method in this research is investigating improvements in solving the non-linearity of the PSD. In addition to the simulation performed in MATALB and PSpice, a model of the system was built to test the applicability of the proposed method. The presented results further confirmed the improvements that was seen in the computer simulation section.

\section{METHODOLOGY}

A semiconductor position sensitive detector (PSD) has been used widely for mark position determination apparatus. Conceptual configuration of PSD and an equivalent pin-cushion PSD circuit is shown in Fig. 1 To better illustrate the function of the PSD, a sectional cut is presented. Fig. 2 shows a sectional cut of a one-dimensional PSD that uses a uniform P-type resistive layer. The 2-D tetra-lateral PSD works based on the same principle too. 


\section{International Journal of Innovative Research in Electrical, Electronics, Instrumentation and Control Engineering}

Vol. 6, Issue 11, November 2018

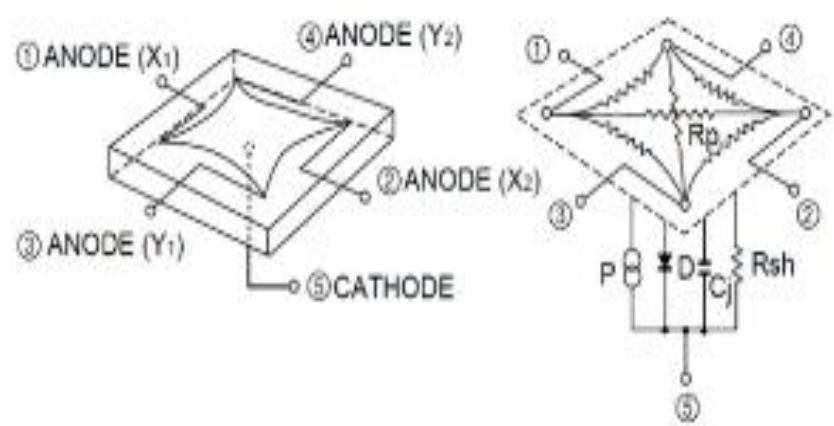

Figure 1. The pin-cushion PSD and its internal circuit.

Photoelectric current flows between the resistance layer and the bias layer at the light incident point, then the output currents are detected from output terminals. By operating the output currents, the image position can be obtained according to the following equation:

$$
X=x w=\frac{\left(I_{b}-I_{a}\right) w}{2\left(I_{a}+I_{b}\right)}
$$

Usually, these operations are performed by adopting an analogue circuit at high speed.

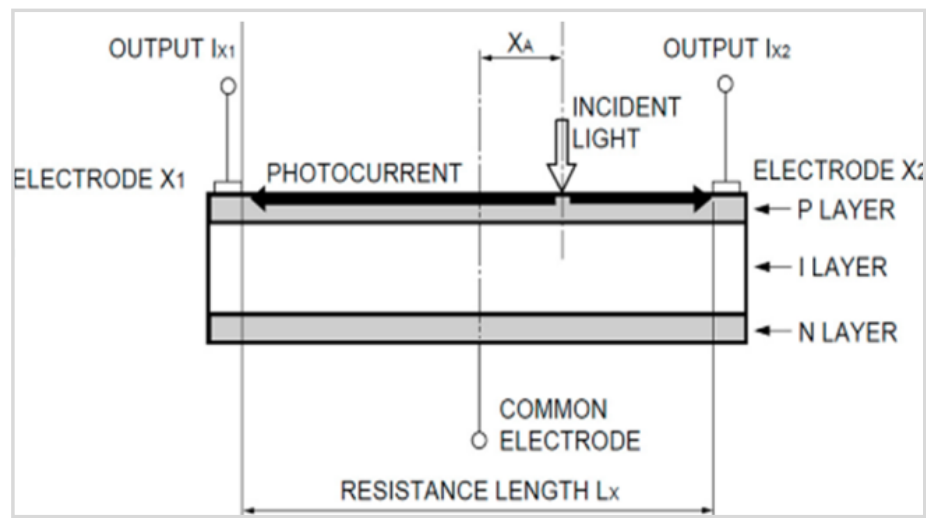

Figure 2. A sectional cut of a one-dimensional PSD is shown here.

The incident light determines the photocurrent that the electrodes receive.

Improvements to mitigate the pincushion-type distortion of the PSD by strengthening the scanning lines of the PSD microscopy system has been achieved by Rahimi et al [16] using the Brown's distortion model [17]. This was also investigated by Villiers [18]

$$
\begin{aligned}
& x=k_{x 1} \cdot \frac{I_{4}-I_{3}}{I_{0}-1.02\left(I_{2}-I_{1}\right)} \cdot \frac{0.7\left(I_{2}+I_{1}\right)+I_{0}}{I_{0}+1.02\left(I_{2}-I_{1}\right)} \\
& y=k_{y 1} \cdot \frac{I_{2}-I_{1}}{I_{0}-1.02\left(I_{4}-I_{3}\right)} \cdot \frac{0.7\left(I_{4}+I_{3}\right)+I_{0}}{I_{0}+1.02\left(I_{4}-I_{3}\right)}
\end{aligned}
$$

The Song method of improving the non-linearity of the is shown in Eq. 2 (2)

The duo lateral PSD uses both the top and bottom surfaces of the diode which gives a good linearity but will cause a more complicated processing and electrical connection to the detector.

In Fig. 3 the equivalent circuit diagram is shown which models the top and bottom resistive layers as two separate variable resistors. The limits for the measurement accuracy are set by the achievable signal to noise ratio (SNR) and the reflector background contrast, defined as the ratio of the powers of the signals received from the reflector and the illuminated background. The former determines the achievable precision and the latter the lower bound for systematic errors. 


\section{International Journal of Innovative Research in Electrical, Electronics, Instrumentation and Control Engineering}

Vol. 6, Issue 11, November 2018

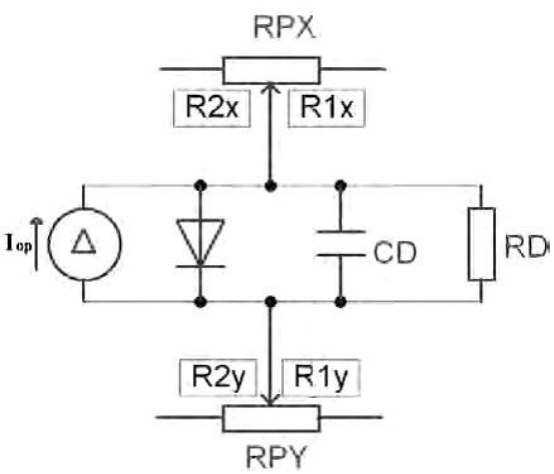

Figure 3. Equivalent circuit diagram of duo-lateral PSD.

III.

SIMULATION RESULTS

The simulation of the proposed system was implemented in MATLAB and PSpice to verify the model system. Fig. 4. shows the overall noise of the PSD system before applying the filtering systems designed in this study. It can be noticed from Fig. 4 that the noise is not Gaussian in effect and have diagonal effects to it. That is why choosing a PSD that does not adhere to the tetra-lateral effect such as a lateral effect PSD is an ideal substitution. This is shown in the postprocessing of the PSD signal [19] and has applications in the robotics localization [20].

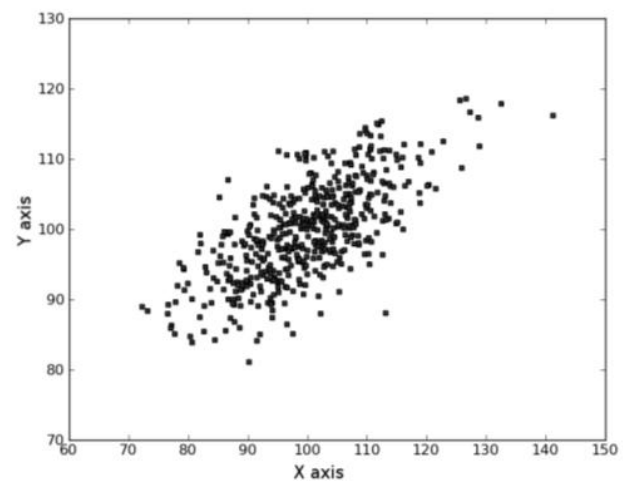

Figure 4. The overall noise associated with the PSD system in the $\mathrm{X}$ and $\mathrm{Y}$ axis of the PSD.

Here the measured precision achieved using CCR method was assessed. The initial result of the simulation is shown in Fig. 5 and 6.
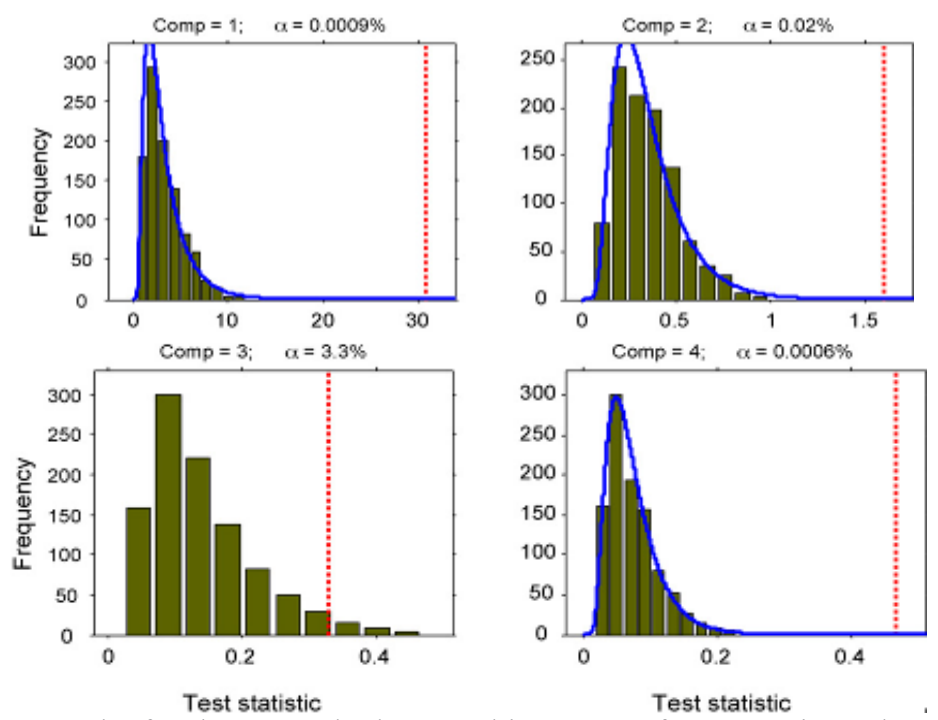

Figure 5. Randomization results for the example data set: histogram of 1000 'noise values', fit using the inverse Gaussian function (the blue line). The red dashed line shows the value under test. 


\section{International Journal of Innovative Research in Electrical, Electronics, Instrumentation and Control Engineering}

Vol. 6, Issue 11, November 2018

The accuracy of the PSD alongside the sigma variable can be improved by

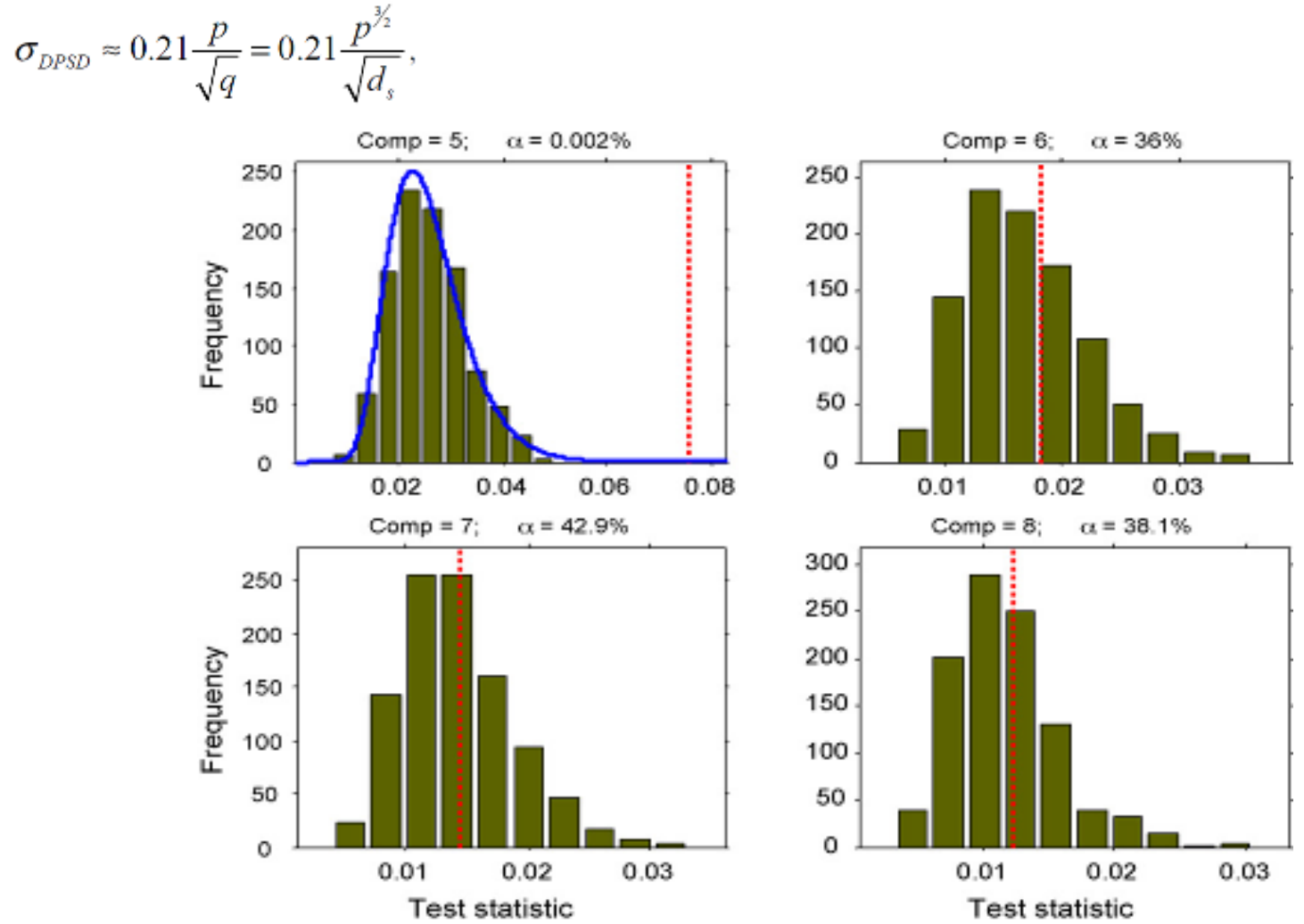

Figure 6. The noise power randomization results for the example data set: histogram of 1000 'noise values', fit using the inverse Gaussian function (the blue line). The red dashed line shows the value under test.

where the spot diameter ds equals qp and $\mathrm{p}$ is the pixel width. The method is reasonably effective up to an accuracy of about $1 / 10$ of a pixel, which is achieved by extending the spot diameter over approximately five pixel widths. When better accuracy is needed, the total number of triggered pixels, and accordingly the total signal energy, rises rapidly, reducing the sensitivity of the PSD. A spectral analysis shows the significant improvement resulting from using this method. A comparison of the improvement is shown in Fig. 7.

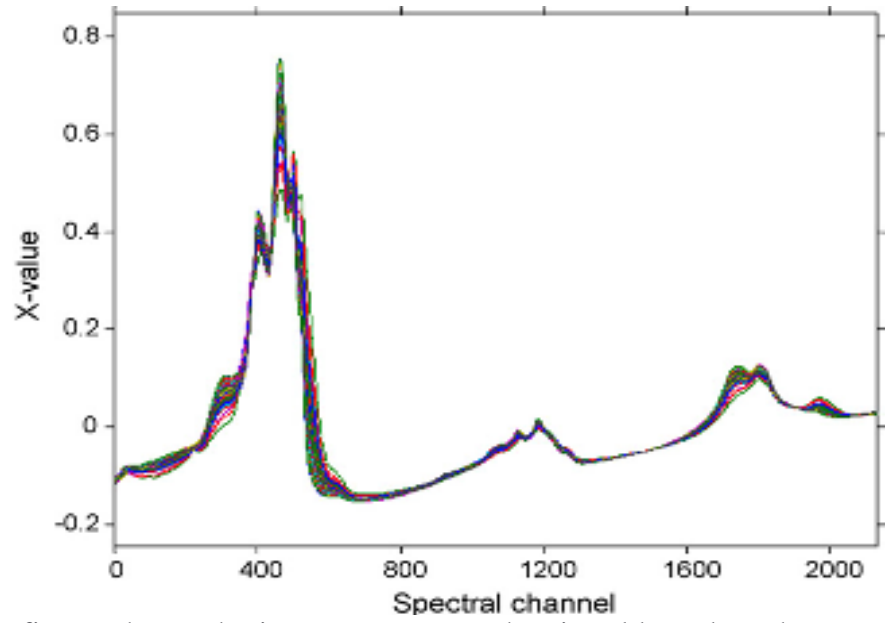

Figure 7. This figure shows the improvement on the signal based on the proposed method.

To further validate this improvement, a sine wave tracking signal was performed using the PSD and the output was processed by the proposed method. The displacement can be significantly improved in this was. This is shown in Fig. 8 . It is shown in the simulation and the actual implementation of the system that the noise can be reduced significantly to improve the precision of the system. 


\section{International Journal of Innovative Research in Electrical, Electronics, Instrumentation and Control Engineering}

Vol. 6, Issue 11, November 2018

\section{CONCLUSION}

This research presents our proposed method of improving the precision of the PSD system. It was shown through the simulation and the actual implementation of the system that the noise associated with overall system was significantly reduced. The main scientific contribution of the paper comprises these modifications the most important of which are the methods and constructions that can be used to improve measurement precision in the performance of a PSD. Overall, the simulation and actual result show a very significant improvement in the precision of the PSD system.
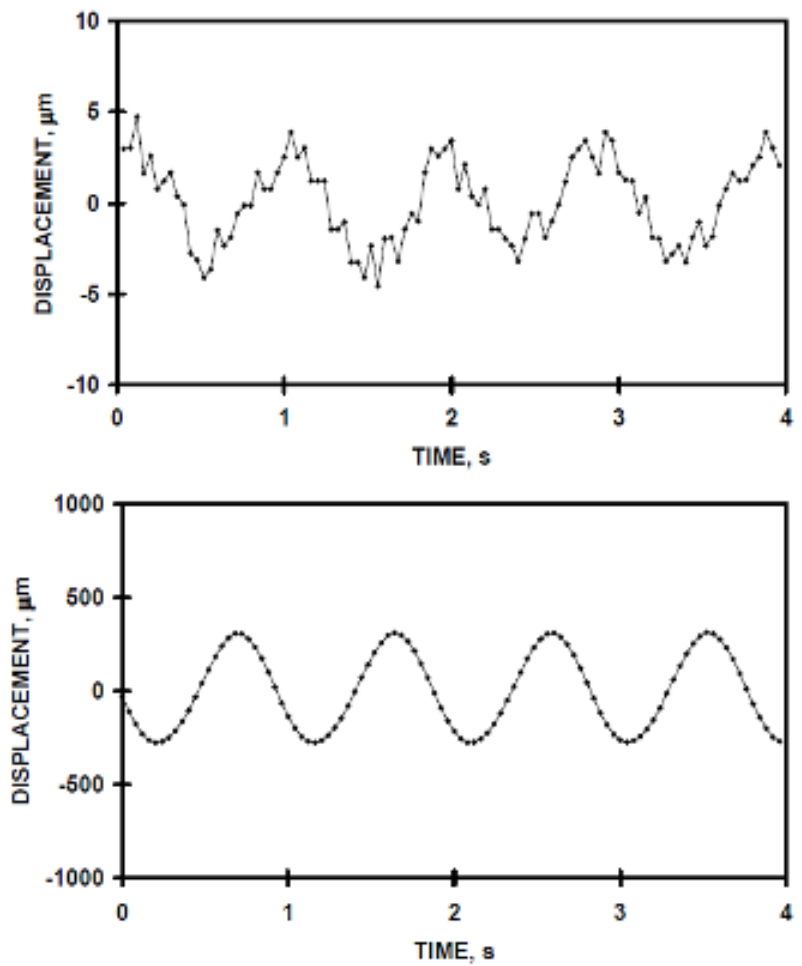

Figure 8. The final improvement of the noise associated with the system is shown and the proposed method has significantly improved the tracking.

\section{ACKNOWLEDGMENTS}

This research was supported by the Singapore Research Program (SRP2018-9741-F2RE). Authors thank the research program of the National University of Singapore.

\section{DECLARATION}

Authors have disclosed no conflicts of interests.

\section{REFERENCES}

[1]. Meyer, E. R. N. S. T. "Atomic force microscopy." Progress in surface science 41, no. 1 (1992): 3-49.

[2]. Mäkynen, Anssi, Tarmo Ruotsalainen, Timo Rahkonen, and Juha Kostamovaara. "CMOS-compatible position-sensitive devices (PSDs) based on photodetector arrays." Sensors and Actuators A: Physical 105, no. 3 (2003): 261-270.

[3]. Chiang, Cheng-Ta, and Yu-Ting Hou. "A CMOS monolithic calibrated position-sensitive detector (PSD) for detecting light-spot position applications." In Mechatronics and Automation (ICMA), 2016 IEEE International Conference on, pp. 862-867. IEEE, 2016.

[4]. Zhuravel, A. P., A. G. Sivakov, O. G. Turutanov, A. N. Omelyanchouk, Steven M. Anlage, A. Lukashenko, A. V. Ustinov, and D. Abraimov. "Laser scanning microscopy of HTS films and devices." Low Temperature Physics 32, no. 6 (2006): $592-607$.

[5]. Rahimi, Mehdi, and Yantao Shen. "PSD microscopy: a new technique for adaptive local scanning of microscale objects." Robotics and biomimetics 4, no. 1 (2017): 6.

[6]. Alford, W. Jerry, R. D. VanderNeut, and Vicent J. Zaleckas. "Laser scanning microscopy." Proceedings of the IEEE 70, no. 6 (1982): 641-651.

[7]. Seyed Mehdi Rahimi. "Towards Developing a Scanning Position Sensitive Detector (PSD) Microscopy: PSD Measurement Enhancement, Adaptive Local Scanning and Implementation." PhD diss., 2016.

[8]. Wallmark, J. Torkel. "A new semiconductor photocell using lateral photoeffect." Proceedings of the IRE 45, no. 4 (1957): $474-483$. 


\section{IJIREEICE \\ International Journal of Innovative Research in Electrical, Electronics, Instrumentation and Control Engineering}

Vol. 6, Issue 11, November 2018

[9]. Pogorelov, A. G., and V. N. Pogorelova. "Quantitative tomography of early mouse embryos: laser scanning microscopy and 3D reconstruction." Journal of microscopy 232, no. 1 (2008): 36-43

[10]. Qian, Dahong, Wanjun Wang, and Ilene J. Busch-Vishniac. "A method for measurement of multiple light spot positions on one positionsensitive detector (PSD)." IEEE transactions on instrumentation and measurement 42, no. 1 (1993): 14-18.

[11]. Rahimi, Mehdi, and Yantao Shen. "Adaptive local scanning: a comprehensive and intelligent method for fast scanning of indiscrete objects." In Multisensor Fusion and Information Integration for Intelligent Systems (MFI), 2014 International Conference on, pp. 1-6. IEEE, 2014.

[12]. M. Rahimi \& Y. Shen. (2016, July). Accelerated adaptive local scanning of complicated micro objects for the PSD scanning microscopy: methods and implementation. In International conference on intelligent autonomous systems (pp. 511-521). Springer, Cham.

[13]. Jung, S.-Y.; Hann, S.; Park, C.-S. TDOA-based optical wireless indoor localization using LED ceiling lamps. IEEE Trans. Consum. Electron. 2011, 57, 1592-1597.

[14]. Rodríguez-Navarro, David, José Luis Lázaro-Galilea, Álvaro De-La-Llana-Calvo, Ignacio Bravo-Muñoz, Alfredo Gardel-Vicente, Georgios Tsirigotis, and Juan Iglesias-Miguel. "Indoor Positioning System Based on a PSD Detector, Precise Positioning of Agents in Motion Using AoA Techniques." Sensors 17, no. 9 (2017): 2124

[15]. Cui, Song, and Yeng Chai Soh. "Linearity indices and linearity improvement of 2-D tetralateral position-sensitive detector." IEEE Transactions on Electron Devices 57, no. 9 (2010): 2310-2316.

[16]. Rahimi, Mehdi, Yudong Luo, Frederick C. Harris, Sergiu M. Dascalu, and Yantao Shen. "Improving measurement accuracy of position sensitive detector (PSD) for a new scanning PSD microscopy system." In Robotics and Biomimetics (ROBIO), 2014 IEEE International Conference on, pp. 1685-1690. IEEE, 2014.

[17]. Brown, D. C. "Decentering Distortion of Lenses." Photometric Engineering 32, no. 3 (1966): 444-462.

[18]. De Villiers, Jason P., F. Wilhelm Leuschner, and Ronelle Geldenhuys. "Centi-pixel accurate real-time inverse distortion correction." In Optomechatronic Technologies 2008, vol. 7266, p. 726611. International Society for Optics and Photonics, 2008.

[19]. Rahimi, Mehdi, and Yantao Shen. "Flash scanning: An ultra fast local scanning of complicated objects for PSD microscopy using 2D bisection." In Real-time Computing and Robotics (RCAR), 2017 IEEE International Conference on, pp. 721-726. IEEE, 2017.

[20]. Park, Jae-Han, Dae-Hee Won, Ki-Young Park, Seung-Ho Baeg, and Moon-Hong Baeg. "Development of a real time locating system using PSD under indoor environments." In SICE-ICASE, 2006. International Joint Conference, pp. 4251-4255. IEEE, 2006 